OPEN ACCESS

Edited by:

Gao-Xia Wei,

Chinese Academy of Sciences, China

Reviewed by:

Weidong $\mathrm{Li}$,

The Ohio State University, USA

Chenglin Zhou,

Shanghai University of Sport, China

*Correspondence:

Ai-Guo Chen

chenaiguo11@sina.com

Heng-Chan Yin

yinhengchan@bnu.edu.cn

Specialty section:

This article was submitted to

Movement Science and Sport

Psychology,

a section of the journal

Frontiers in Psychology

Received: 31 July 2016 Accepted: 01 November 2016 Published: 21 November 2016

Citation:

Chen A-G, Zhu L-N, Yan J and

Yin H-C (2016) Neural Basis of Working Memory Enhancement after Acute Aerobic Exercise: $\mathrm{fMR}$ Study of Preadolescent Children.

Front. Psychol. 7:1804

doi: 10.3389/fpsyg.2016.01804

\section{Neural Basis of Working Memory Enhancement after Acute Aerobic Exercise: fMRI Study of Preadolescent Children}

\author{
Ai-Guo Chen ${ }^{1 *}$, Li-Na Zhu', Jun Yan ${ }^{1}$ and Heng-Chan Yin ${ }^{2 *}$ \\ ${ }^{1}$ College of Physical Education, Yangzhou University, Yangzhou, China, ${ }^{2}$ School of Physical Education and Sports Science, \\ Beijing Normal University, Beijing, China
}

Working memory lies at the core of cognitive function and plays a crucial role in children's learning, reasoning, problem solving, and intellectual activity. Behavioral findings have suggested that acute aerobic exercise improves children's working memory; however, there is still very little knowledge about whether a single session of aerobic exercise can alter working memory's brain activation patterns, as assessed by functional magnetic resonance imaging (fMRl). Therefore, we investigated the effect of acute moderate-intensity aerobic exercise on working memory and its brain activation patterns in preadolescent children, and further explored the neural basis of acute aerobic exercise on working memory in these children. We used a within-subjects design with a counterbalanced order. Nine healthy, right-handed children were scanned with a Siemens MAGNETOM Trio 3.0 Tesla magnetic resonance imaging scanner while they performed a working memory task ( $\mathrm{N}$-back task), following a baseline session and a 30-min, moderate-intensity exercise session. Compared with the baseline session, acute moderate-intensity aerobic exercise benefitted performance in the N-back task, increasing brain activities of bilateral parietal cortices, left hippocampus, and the bilateral cerebellum. These data extend the current knowledge by indicating that acute aerobic exercise enhances children's working memory, and the neural basis may be related to changes in the working memory's brain activation patterns elicited by acute aerobic exercise.

Keywords: acute aerobic exercise, working memory, N-back, brain activation patterns, fMRI, preadolescent children

\section{INTRODUCTION}

Working memory involves temporary storage and manipulation of information assumed necessary for a wide range of complex cognitive activities (Baddeley, 1992, 2003). It is an essential element for learning, memory, decision-making, cognitive control, other high-level cognitive activities, and brain development (Baddeley, 1992, 2003; Ericsson and Kintsch, 1995; Bechara et al., 1998; de Jong, 1998; Passolunghi and Siegel, 2001; Swanson and Sachse-Lee, 2001; Bull et al., 2008). Deficits in working memory will seriously harm the development of children's physical, mental, and social achievements; conversely, individuals, local communities, and society will benefit from well-developed working memory (Siegel and Ryan, 1989; Ericsson and Kintsch, 1995; Bechara et al., 1998; de Jong, 1998; Passolunghi and Siegel, 2001; Bull et al., 2008). Although working memory has a neural 
anatomical basis, it is flexible and plastic, and thus, can be improved through training, especially in high correlation with children's cognitive development (Olesen et al., 2004; Westerberg and Klingberg, 2007; Takeuchi et al., 2010; Kamijo et al., 2011; Mrazek et al., 2013). Therefore, working memory has attracted increasing research attention in various fields and has become a frontier in interdisciplinary research. Identification of effective methods to develop children's working memory is a focus of the current research.

A burgeoning body of literature has emerged on exercise's positive effects on the brain and cognition. Aerobic exercise as an effective method for improving children's brain and cognitive function has been gradually recognized and practiced (Davis et al., 2007, 2011; Erickson and Kramer, 2009; Diamond and Lee, 2011; Erickson et al., 2011; Chang et al., 2012, 2013; Chapman et al., 2013; Hötting and Röder, 2013; Verburgh et al., 2013; Chen et al., 2014). Recent findings have also suggested that acute aerobic exercise enhances working memory (Pontifex et al., 2009; McMorris et al., 2011; Li et al., 2014). Nevertheless, it is not clear whether the neural basis of improvement in children's working memory is elicited by acute aerobic exercise.

Functional magnetic resonance imaging (fMRI) noninvasively and safely measures and maps brain activity (Fox and Raichle, 2007). With fMRI, brain activation can be evaluated by measuring the blood oxygenation level-dependent (BOLD) contrast signal, which reflects a change in the ratio of oxygenated to deoxygenated hemoglobin that occurs with brain activation and increases in local blood volume. In a growing number of studies, fMRI is applied to directly understand how brain function changes with aerobic exercise or training (Kramer and Erickson, 2007; Chaddock et al., 2010; Davis et al., 2011; Erickson et al., 2011; Li et al., 2014). Through fMRI, working memory's neural basis has been found to have a specific pattern of brain activation, which, in working memory, mainly includes frontal and parietal cortices (Cohen et al., 1997; Klingberg et al., 2002; Baddeley, 2003; Klingberg, 2006; Darki and Klingberg, 2014; Ester et al., 2015; Harding et al., 2015). These brain regions' functional specialization and cooperation are the operating basis of working memory (Diwadkar et al., 2000; Baddeley, 2003). Moreover, studies from cognitive psychology and neuroscience have revealed that working memory training increases and decreases in task-related BOLD activity in different regions associated with increases in working memory capacity (Constantinidis and Klingberg, 2016). That is, both increases and decreases in the BOLD signal can be informative about the stimulus maintained in working memory, reflecting excitatory and suppressive responses to stimuli's orientation and motion. Additionally, a decrease in the BOLD signal in a certain area is often interpreted as an increase in the area's "efficiency" in performing its function. In short, improvement in working memory relates to its activation pattern changes (Klingberg et al., 2002; Olesen et al., 2004; Westerberg and Klingberg, 2007; Takeuchi et al., 2010; Kamijo et al., 2011; Mrazek et al., 2013). Therefore, the key to clarifying the neural basis of working memory enhancement caused by children's acute aerobic exercise is to reveal changes in working memory's brain activation patterns elicited by acute aerobic exercise.
In summary, the present study explores the effect of acute moderate-intensity aerobic exercise on working memory and its brain activation patterns in preadolescent children, and further explored the neural basis of acute aerobic exercise on working memory in these children. We hypothesize that our study would both replicate previous studies, demonstrating that acute aerobic exercise improves working memory, and extend our current understanding of this process to discover that acute aerobic exercise can better optimize working memory's brain activation pattern.

\section{MATERIALS AND METHODS}

\section{Ethic Statement}

The study protocol was approved by the Institutional Review Board of Beijing Normal University. All participants and their guardians provided written consent, and the protocol was approved by the institutional review board of Beijing Normal University.

\section{Participants}

Nine healthy children in fifth grade (10 years old; five males, four females) were recruited through primary school advertising. They had normal or corrected-to-normal vision and were righthanded, as assessed by the Edinburgh Test (Oldfield, 1971). They also completed a set of questions about history of drug abuse or inherited disease and general intelligence (Wechsler Intelligence Scale for Children-IV-Chinese Version, WISC-IV-C) (Zhang, 2009). Exclusions included any medical condition that would limit physical activity or affect study results (including neurological or psychiatric disorders). The study was conducted in accordance with the Declaration of Helsinki. The study protocol was approved by the Institutional Review Board of the Brain Imaging Center of the State Key Laboratory of Cognitive Neuroscience and Learning, Beijing Normal University. Written informed consent was obtained from all participants and their legal guardians after experimental procedures were fully explained.

\section{Acute Aerobic Exercise Protocol}

Exercise was performed on a bicycle ergometer (MONARK 834, Sweden) with moderate intensity, which has been shown to benefit children's cognition (Hillman et al., 2009; Chen et al., 2014). This study used $60-69 \%$ of the predicted maximal heart rate, defined as 220 minus age in years, to determine the exercise intensity target heart rate (American College of Sports Medicine, 2006). Participants spent 2 min cycling to warm up, $30 \mathrm{~min}$ exercising at moderate intensity, and finally, 3 min cooling down at a self-determined pace. Heart rate was monitored in real time using a Polar heart rate monitor (RS800XSD, Finland) throughout the acute aerobic exercise protocol, which was led by the same instructor, one-on-one.

\section{Working Memory Task}

An N-back task (Smith and Jonides, 1997; Owen et al., 2005) was programmed using E-Prime to assess working memory. 

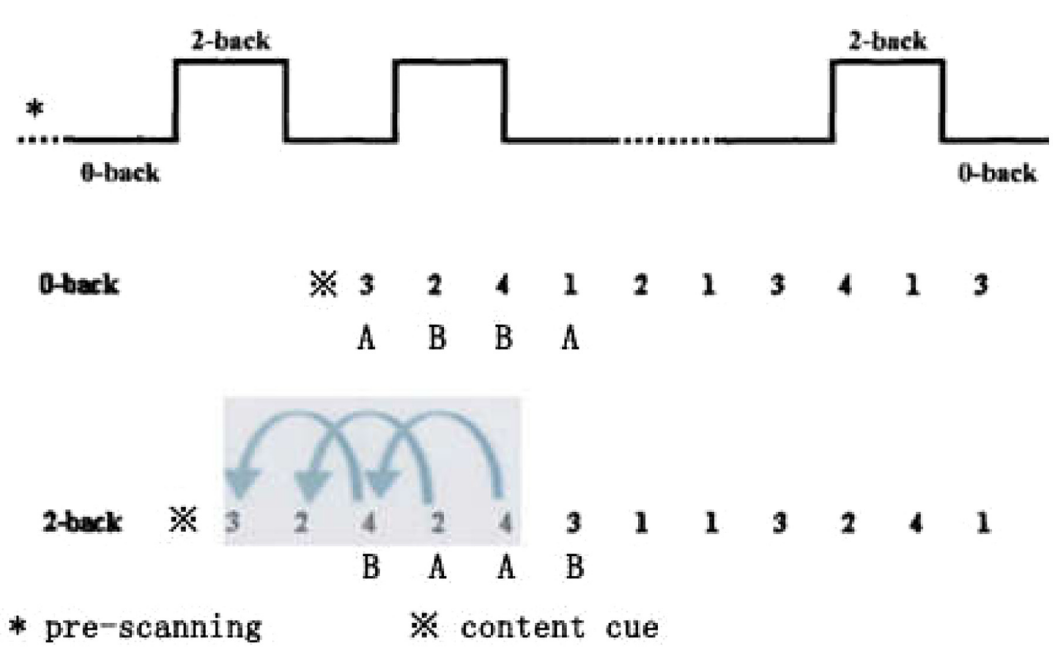

FIGURE 1 | Design of the N-back working memory task.

The task used group block design (Figure 1), contained 0and 2-back conditions, and was performed alternatingly. The 2-back condition was a working memory task, while the 0-back condition was a control task that did not need to manipulate working memory. Thus, the contrast between 0 -back and 2 -back conditions reflects, to a large degree, time-related aspects of processing stimuli in working memory. The N-back task had five 2-back conditions and six 0 -back conditions. The task consisted of a series of changing number stimuli displayed at the center of a computer screen (i.e., 1, 2, 3, 4). The first block of three phases was pre-scanning. Each block of one to four phases was adaptive, and the screen center was "ready," prompting the participant to prepare to respond. Each stimulus was displayed on the screen for 2,000 ms and each phase's duration was 2,000 ms. The presentation time for each number stimulus was $1,500 \mathrm{~ms}$, and the reaction time was $500 \mathrm{~ms}$. Working memory's behavioral performance consisted of mean reaction time (RT) and mean accuracy (Acc); consequently, the shorter the RT, and/or the higher the Acc, the better the participant's working memory.

Each 0-back condition included 11 phases in random order. The first phase was an experimental content cue: single or double; when the numeral 1 or 3 appeared, pressing button $A$; when the numeral 2 or 4 appeared, pressing button $B$. These four numbers were displayed randomly at equal probability.

Each 2-back condition included 13 phases in random order. The first phase was an experimental content cue: Remember and go back 2; the participant needed to remember the second and third stimuli in order of appearance. When the fourth stimulus appeared, they had to respond whether it was the same as the second; if it was, they pressed $A$, and otherwise, pressed $B$ on the keyboard with both hands.

\section{Experimental Procedure}

This experiment had a completely within-subjects design. It was conducted in the Imaging Center for Brain Research at Beijing Normal University. During the first visit, participants and their legal guardians completed all paperwork, including written informed assent/consent, as previously described. Following paperwork completion, an N-back task practice was administered to each participant, and the experimenter checked their performances to ensure that the participants understood the task. If a participant's task performance was below $80 \%$ (Acc), the same practice was re-administered. In the formal experiment, all participants attended two sessions (i.e., baseline and exercise), with the order counterbalanced across participants at the same time on two separate days (a 7-day interval) in which they had not participated in physical education or other structured physical activity. So half participants received the baseline session on the first day and the exercise session on the second day. The other half received the exercise session on the first day and the baseline session on the second day. The baseline session consisted of $30 \mathrm{~min}$ of seated rest, during which all participants were fitted with a heart rate monitor and their resting heart rates were recorded. Following the seated rest period, participants completed an N-back task during MRI scanning. The exercise session consisted of a 30-min rest, with the resting heart rate recorded, and an acute aerobic exercise protocol during which HR was recorded in real time. Following the completion of the acute aerobic exercise protocol, once participants' HRs returned to within $10 \%$ of their resting heart rate levels, the N-back task was performed during MRI scanning. Upon completion of both sessions, participants and their legal guardians received fair remuneration for their involvement in the experiment.

\section{Functional MRI Data Acquisition and Image Processing}

Participants underwent one scan for high-resolution structural images of the whole brain on a 3T Siemens Magnetom Trio system (Siemens) with total imaging matrix in the Imaging Center for Brain Research, Beijing Normal University. Functional images were obtained using an echo-planar imaging (EPI) sequence, with the following scan parameters: $\mathrm{TR}=2,000 \mathrm{~ms}$, 
$\mathrm{TE}=30 \mathrm{~ms}$, gap $=1 \mathrm{~mm}$, flip angle $(\mathrm{FA})=90^{\circ}$, slice thickness $=3.0 \mathrm{~mm}$, field of view $(\mathrm{FOV})=200 \times 200 \mathrm{~mm}$, and inplane resolution $=64 \times 64$. Resulting data included 148 brain volumes with 33 axial slices. During the fMRI scans, all participants were instructed to stay relaxed and move as little as possible. High-resolution structural images were acquired using a magnetization-prepared rapid gradient echo, three-dimensional T1-weighted sequence ( $\mathrm{TR}=2000 \mathrm{~ms}$, $\mathrm{TE}=3.39 \mathrm{~ms}, \mathrm{~T} 1=1100 \mathrm{~ms}, \mathrm{FA}=7^{\circ}$, thickness $=1.33 \mathrm{~mm}$, $\mathrm{FOV}=200 \times 200 \mathrm{~mm}$, acquisition matrix $=256 \times 256)$.

Functional image preprocessing and statistical analyses were conducted with $\mathrm{DPABI}{ }^{1}$, based on $\mathrm{SPM} 8^{2}$. The first three volumes of functional images were discarded for signal equilibrium and participants' adaptation to scanning noise. Subsequent functional images underwent the following preprocessing steps: slice-timing correction, realignment, co-registration, and New Segment + Diffeomorphic Anatomical Registration Through Exponentiated Liealgebra (DARTEL) with high-resolution structural scans (Ashburner and Friston, 2005). The DARTEL tool (Ashburner, 2007) was used to compute transformations from individual native space to Montreal Neurological Institute (MNI) coordinate space. Then, the segmented BOLD volumes were normalized into standardized MNI space using the DARTEL template and resampled to $3 \mathrm{~mm} \times 3 \mathrm{~mm} \times 3 \mathrm{~mm}$ isotropic voxels. Finally, normalized images were smoothed with an $8 \mathrm{~mm} \times 8 \mathrm{~mm} \times 8 \mathrm{~mm}$ full width at half maximum Gaussian kernel.

\section{Statistical Analysis}

First, descriptive data were evaluated to determine the appropriateness of exercise intensity manipulation. Second, behavioral improvements in RT and Acc for working memory across the two sessions were analyzed by paired $t$-test with SPSS. Third, functional changes were analyzed with two procedures, individual and group analysis. Individual analysis: The 0-back condition was considered a control task in the present study. The 2-back condition required maintenance and permanent update of relevant pieces of information in working memory. Then, statistical parametric maps (SPMs) were computed for individual participants by using the general linear model (GLM) with separate hemodynamic basis response function modeling MR signal responses for each task period. Contrast images (2-back vs. 0-back) on estimates of interest were obtained for each participant. Group analysis: There were two steps for group analysis based on the contrast images (2-back vs. 0-back). The first was to identify whether the N-back task is successfully inducing the common brain activation patterns of working memory for each session (baseline and exercise), we conducted a one-sample $t$-test on the contrast images (2-back vs. 0 -back). The second was to examine neural activation differences between two sessions (baseline and exercise); we conducted a paired $t$-test on the contrast images (2-back vs. 0 -back) to detect acute aerobic exercise gains.

\footnotetext{
${ }^{1}$ http://rfmri.org/dpabi

${ }^{2}$ http://www.fil.ion.ucl.ac.uk/spm/software/spm8/
}

\section{RESULTS}

\section{Exercise Intensity Manipulation}

The heart rates for the baseline and exercise sessions were 41.80 and $64.52 \%$, respectively, of the predicted maximal heart rate $[t(8)=23.70, p<0.001]$. The two sessions' differing heart rates and percentages of the predicted maximal heart rate suggest that the selected moderate-intensity exercise was appropriate.

\section{Behavioral Performances}

Table 1 presents detailed behavioral measures based on the two sessions and the N-back conditions. To explore behavioral performance differences between the baseline and exercise sessions, a paired $t$-test was used on RT and accuracy. There were no significant differences between the baseline and exercise sessions on 0 -back RT $[t(8)=1.37, p>0.05$ and Acc $t(8)=-1.03, p>0.05 ; 2$-back Acc $t(8)=-0.82, p>0.05]$. The 2back RT of the exercise session showed significant improvement after acute exercise $t(9)=2.79, p<0.05, r^{2} \mathrm{pb}=0.49$; that is, shorter RT demonstrated better working memory.

\section{Brain Activation Differences}

The first analytical goal was to examine working memory activation in two scanning sessions (baseline and exercise) with contrast images (2-back vs. 0-back), calculated as an assessment of the dynamic range of neural differences activation between a control task and a working memory task. The statistical threshold was set at $p<0.001$ with a cluster size threshold of 75 voxels, which is equivalent to cluster-level $p<0.05$, AphaSim corrected. Specifically, in the baseline session, regions of significant BOLD activation were the left Superior Frontal Gyrus (SFG), bilateral Middle Frontal Gyrus (MFG), right Inferior Frontal Gyrus (IFG), bilateral Parahippocampa gyrus (PHP), right Middle Occipital Gyrus (MOG), left Superior Temporal Gyrus (STG), and bilateral Cerebellum Posterior Lobe; in the exercise session, right Medial Frontal Gyrus (MEDFG), MFG, left Superior Parietal Lobule (SPL), right Inferior Parietal Lobule (IPL), right Superior Occipital Gyrus (SOG), left Anterior Cingulate Cortex (ACC), right Posterior Cingulate Cortex (PCC), and bilateral Cerebellum Posterior Lobe were activated (Table 2; Figure 2). This analysis of activated brain regions revealed the common patterns of working memory.

In further comparisons of brain regions' activated changes between the baseline and exercise sessions, a paired $t$-test revealed significant difference. The statistical threshold was set at $p<0.025$ with a cluster size threshold of 100 voxels, which is equivalent to cluster-level $p<0.05$,

TABLE 1 | Children's behavioral performances on $\mathrm{N}$-back task in baseline and exercise sessions.

\begin{tabular}{lcclcc}
\hline \multirow{2}{*}{ Condition } & \multicolumn{2}{c}{ Baseline session } & & \multicolumn{2}{c}{ Exercise session } \\
\cline { 2 - 3 } \cline { 5 - 6 } & ACC (\%) & RT(ms) & & ACC (\%) & RT(ms) \\
\hline 0-back & $0.95 \pm 0.03$ & $682.80 \pm 52.68$ & & $0.96 \pm 0.03$ & $643.92 \pm 73.35$ \\
2-back & $0.81 \pm 0.03$ & $963.13 \pm 125.24$ & & $0.82 \pm 0.03$ & $877.57 \pm 53.74$
\end{tabular}


TABLE 2 | Children's brain activation patterns during working memory in baseline and exercise scanning sessions.

\begin{tabular}{|c|c|c|c|c|c|c|}
\hline \multirow[t]{2}{*}{ Primary regions } & \multirow[t]{2}{*}{ Brodmann area } & \multirow[t]{2}{*}{ Size (voxel) } & \multirow[t]{2}{*}{ Max T-statistic } & \multicolumn{3}{|c|}{ MNI coordinates } \\
\hline & & & & $x$ & $y$ & $z$ \\
\hline \multicolumn{7}{|l|}{ Baseline session } \\
\hline B_Cerebellum Posterior Lobe & - & 103 & 21.84 & -36 & -60 & -45 \\
\hline L_Parahippocampa Gyrus & 30 & 235 & -13.23 & -24 & -21 & -18 \\
\hline R_Parahippocampa Gyrus & 34 & 4249 & -21.29 & 24 & -3 & -15 \\
\hline L_Medial Frontal Gyrus & 10 & 506 & -8.62 & -3 & 54 & -6 \\
\hline R_Middle Occipital Gyrus & 18 & 1352 & -19.21 & 21 & -99 & 3 \\
\hline L_Superior Temporal Gyrus & 48 & 289 & -13.35 & -39 & -15 & 15 \\
\hline R_Inferior Frontal Gyrus & $47 / 48$ & 158 & 17.06 & 30 & 18 & -9 \\
\hline B_Middle Frontal Gyrus & $32 / 24$ & 844 & 17.54 & 0 & 18 & 42 \\
\hline L_Superior Frontal Gyrus & 9 & 108 & -9.41 & -9 & 48 & 42 \\
\hline \multicolumn{7}{|l|}{ Exercise session } \\
\hline L_Cerebellum Posterior Lobe & - & 111 & 18.25 & -33 & -66 & -48 \\
\hline B_Cerebellum Posterior Lobe & - & 80 & 9.59 & -6 & -81 & -30 \\
\hline L_Middle Frontal Gyrus & 47 & 84 & -9.48 & -36 & 39 & -18 \\
\hline L_Anterior Cingulate Cortex & 25 & 131 & -9.72 & -6 & 24 & -6 \\
\hline R_Medial Frontal Gyrus & 10 & 371 & -15.92 & 6 & 63 & -3 \\
\hline R_Middle Frontal Gyrus & 46 & 246 & 11.51 & 45 & 48 & 15 \\
\hline R_Superior Occipital Gyrus & 19 & 160 & -9.06 & 33 & -84 & 24 \\
\hline R_Posterior Cingulate Cortex & 30 & 442 & -9.49 & 6 & -51 & 21 \\
\hline L_Middle Frontal Gyrus & 32 & 1015 & 34.98 & -6 & 21 & 39 \\
\hline L_Superior Parietal Lobule & 7 & 320 & 11.94 & -6 & -72 & 57 \\
\hline R_Inferior Parietal Lobule & 40 & 215 & 14.84 & 48 & -42 & 39 \\
\hline
\end{tabular}

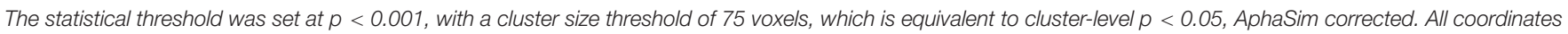
are reported in the MNI format.

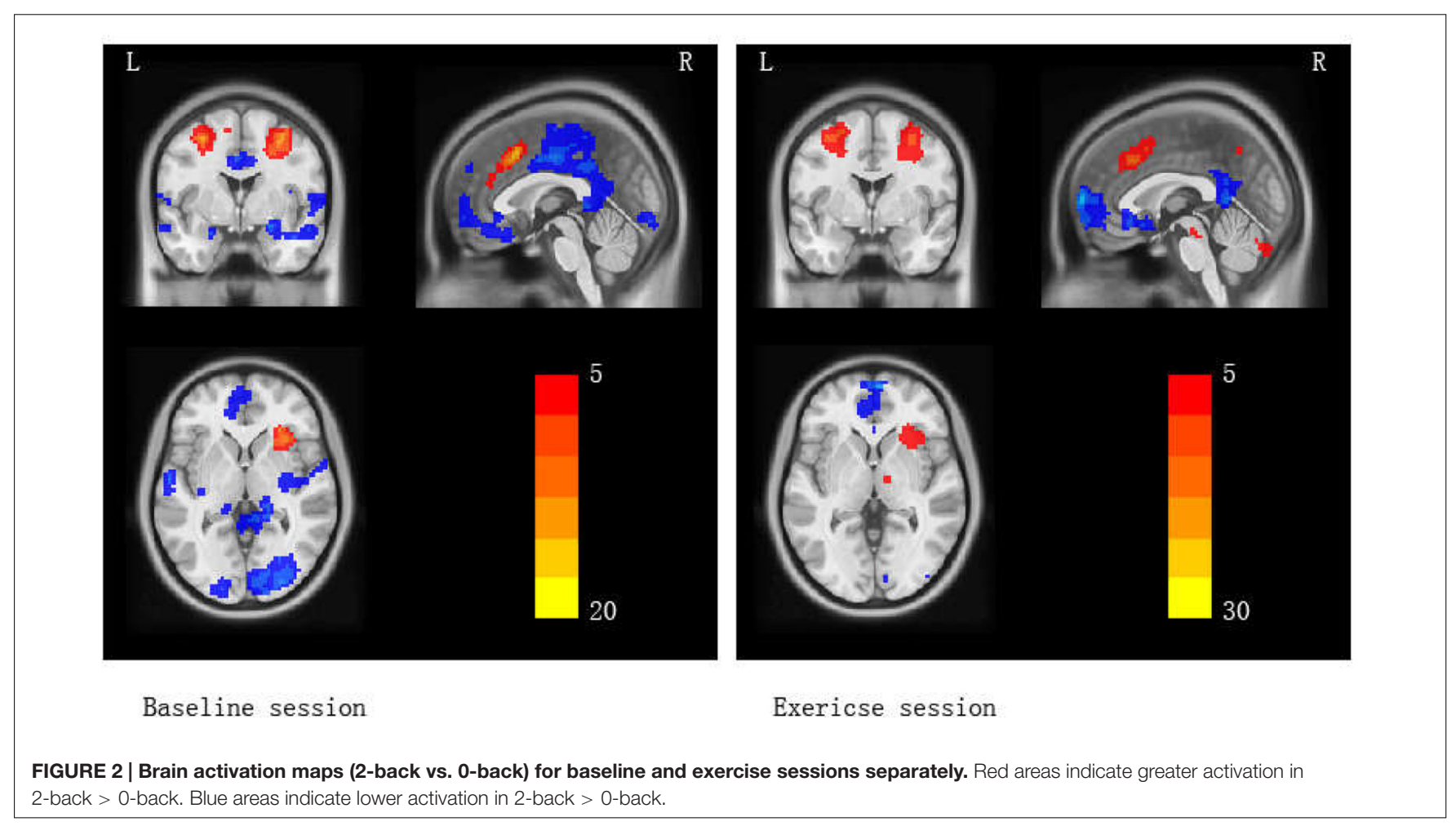


TABLE 3 | Significant brain activation of paired $t$-test between children's baseline and exercise MRI scanning.

\begin{tabular}{|c|c|c|c|c|c|c|}
\hline \multirow[t]{2}{*}{ Primary regions } & \multirow[t]{2}{*}{ Brodmann area } & \multirow[t]{2}{*}{ Size (voxel) } & \multirow[t]{2}{*}{ Max T- statistic } & \multicolumn{3}{|c|}{ MNI coordinates } \\
\hline & & & & $x$ & $y$ & $z$ \\
\hline L_Superior/Inferior Parietal Lobule & $7 / 40$ & 497 & 8.64 & -15 & -57 & 45 \\
\hline R_Superior Parietal Lobule & $5 / 7$ & 191 & 6.57 & 12 & -60 & 54 \\
\hline L_Hippocampus & 20 & 208 & 8.23 & -30 & -30 & -12 \\
\hline L_Cerebellum & - & 347 & 7.18 & -24 & -60 & -33 \\
\hline R_Cerebellum & - & 108 & 6.47 & 36 & -63 & -27 \\
\hline
\end{tabular}

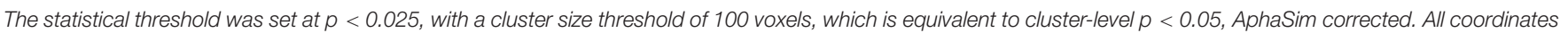
are reported in the MNI format.

AphaSim corrected. Specifically, the exercise session resulted in greater activation of SPL, IPL, left Hippocampus (HIP), and bilateral Cerebellum (Table 3; Figure 3). This analysis indicated that acute aerobic exercise significantly increased some regions of brain activities for working memory.

\section{DISCUSSION}

This study investigated behavioral and neural effects of children's acute aerobic exercise on a working memory task. In both baseline and exercise sessions, participants were tested with a working memory task while being scanned. The exercise session, an acute aerobic exercise protocol, was compared to a baseline session without exercise intervention. Consequently, reliable acute aerobic exercise gains emerged, allowing us to test for acute aerobic exercise effects on the two sessions.

\section{Behavioral Performances}

A rapidly growing body of literature indicates that, from both behavioral and neuroelectric perspectives, acute aerobic exercise improves working memory (Lardon and Polich, 1996; Hillman et al., 2008; Erickson and Kramer, 2009; Chang et al., 2012, 2013; Chen et al., 2014; Drollette et al., 2014). As observed here, children's working memory performance in the exercise session was better than in the baseline session-in agreement with previous studies. Accordingly, the present behavior results have been again verified: acute aerobic exercise beneficially impacts children's working memory.

\section{Brain Activation Patterns of Working Memory}

To our knowledge, many previous studies have explored the macroscale of working memory's neural system. First, in an $\mathrm{N}$-back task, the present study successfully induced working memory's common brain activation patterns. However, how

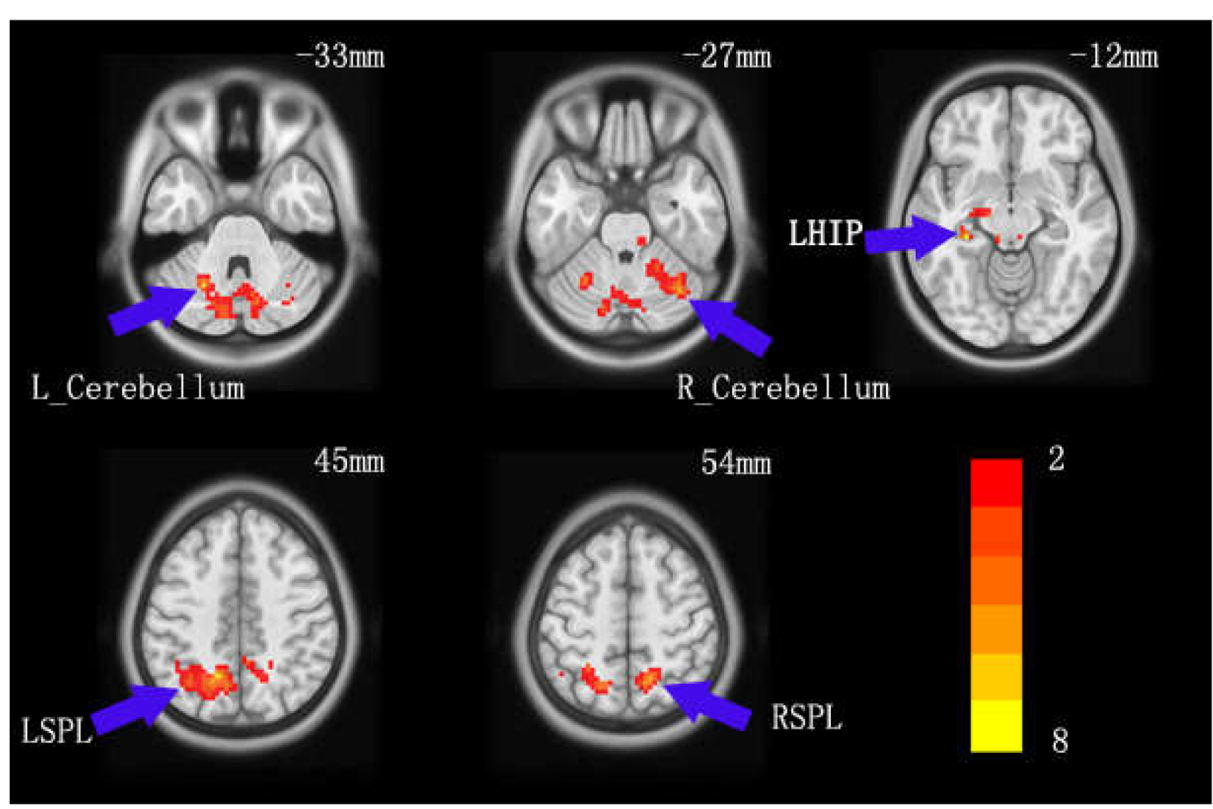

FIGURE 3 | The change in children's brain activation pattern of working memory caused by acute aerobic exercise. Red areas indicate higher activation in the exercise session relative to the baseline session. 
do working memory's brain activation patterns run? Cognitive neuropsychology observations concluded that working memory's neural basis is quite widespread, including the cerebral cortex (e.g., frontal, temporal, parietal, and occipital lobes) and subcortical areas (e.g., thalamus, amygdala, hippocampus, and cerebellum) (LaBar et al., 1999; Smith and Jonides, 1999; Diwadkar et al., 2000; Baddeley, 2003; Osaka et al., 2004; Owen et al., 2005). Brain areas in our results matched these and indicated that the $\mathrm{N}$-back task successfully induced working memory activation patterns.

Second, we compared the exercise session's brain activation maps to those of the baseline session. Regarding acute aerobic exercise-related neural effects, the main findings were that acute aerobic exercise raised working memory's brain activation patterns in bilateral SPL, left IPL, left HIP, and bilateral cerebellum. These results are fully consistent with our prediction that acute exercise significantly influenced working memory's brain activities. These results may be of great importance for understanding the neural basis of acute aerobic exercise's effect on working memory. Indeed, prior studies have demonstrated that improved cognitive function results from brain plasticity changes caused by aerobic exercise (Kramer and Erickson, 2007; Erickson and Kramer, 2009; Voss et al., 2013). One finding reported that high-fitness children showed greater bilateral hippocampal volumes, positively associated with performance on memory task (Chaddock et al., 2010); this study first suggested that aerobic fitness can impact the structure and function of the developing human brain. Similarly, a recent study suggested that significant changes after acute aerobic exercise with brain activation reflected improved working memory in young female college students (Li et al., 2014). Thus, acute aerobic exercise could benefit children's working memory at a macro-neural level.

What is the meaning of children's greater brain activation caused by acute aerobic exercise? Several previous studies have addressed this question, assisting us to understand these increases in SPL, IPL, and bilateral cerebellum and hippocampus. Development of functionality in these areas plays an important role in cognitive development during childhood, and parietal cortices are known to be involved in working memory (Jonides et al., 1998; Culham and Kanwisher, 2001; Koenigs et al., 2009). Working memory capacity significantly correlated with brain activities in the parietal gyrus (Vogel and Machizawa, 2004; Todd and Marois, 2005). Similarly, another study reported that higher parietal activity was associated with higher working memory capacity in children (Klingberg et al., 2002). A meta-analysis concluded that the functional topography of the cerebellum is particularly involved in sensorimotor, language, spatial, and working memory (Stoodley and Schmahmann, 2009). Prior evidence indicated that cognitive training could increase neural activity in cerebellar circuits in children with ADHD (Hoekzema et al., 2010). Recent studies of the cerebellum have revealed connections between the cerebellum and parietal gyrus that might support cerebellar contributions to working memory (Allen et al., 2005). The hippocampus plays an important role in the formation of new memories about experienced events (Vinogradova, 2001). Moreover, previous observations found that the parietal lobule is the target of disynaptic output from two subcortical sites, in which the parietal lobule receives a strong disynaptic input from the hippocampus and the cerebellum contains a distinct output channel that targets a portion of the parietal lobule (Clower et al., 2001). At this point, we speculate on the neural basis of improved working memory induced by acute aerobic exercise: Greater activation of the cerebellum might contribute to the parietal cortex's better functioning and strengthened correlations between the hippocampus and parietal cortex, consequently enhancing children's working memory.

This study is not without its limitations. First, a completely within-subjects design was used in current study in which all subjects are exposed to every experimental session. The drawback of this design is the absence of control group. However, this design ensures that every subject acts at their own control, so there are few problems with matching age, gender, and lifestyle, reducing the chances of confounding factors. Second, generalization of our results is limited by small sample size. Future studies with a larger sample may be more amenable to investigate the effect of acute moderate-intensity aerobic exercise on working memory and its brain activation patterns in preadolescent children.

\section{CONCLUSION}

Here, we suggest that acute aerobic exercise results in children's greater cognitive gains. These data extended the current knowledge base by indicating that acute aerobic exercise enhances children's working memory, in which the neural basis may be related to changes in working memory's brain activation patterns elicited by the exercise.

\section{AUTHOR CONTRIBUTIONS}

A-GC and H-CY designed the study and oversaw the data collection. L-NZ and A-GC analyzed the data and wrote the initial manuscript. JY and H-CY assisted with data analysis and organized the manuscript. All authors played a part in the manuscript's preparation at each stage of its development. All authors have read and approved the manuscript's final version.

\section{ACKNOWLEDGMENTS}

This research was supported in part by grants from the National Natural Science Foundation of China (31300863) and the Fok Ying Tung Education Foundation (141113) to AGC. 


\section{REFERENCES}

Allen, G., McColl, R., Barnard, H., Ringe, W. K., Fleckenstein, J., and Cullum, C. M. (2005). Magnetic resonance imaging of cerebellar-prefrontal and cerebellar-parietal functional connectivity. Neuroimage 28, 39-48. doi: 10.1016/j.neuroimage.2005.06.013

American College of Sports Medicine (2006). ACSM's Guidelines for Exercise Testing and Prescription, 7th Edn. New York, NY: Lippincott Williams and Wilkins.

Ashburner, J. (2007). A fast diffeomorphic image registration algorithm. Neuroimage 38, 95-113. doi: 10.1016/j.neuroimage.2007.07.007

Ashburner, J., and Friston, K. J. (2005). Unified segmentation. Neuroimage 26, 839-851. doi: 10.1016/j.neuroimage.2005.02.018

Baddeley, A. (1992). Working memory: the interface between memory and cognition. J. Cogn. Neurosci. 4, 281-288. doi: 10.1162/jocn.1992.4.3.281

Baddeley, A. (2003). Working memory: looking back and looking forward. Nat. Rev. Neurosci. 4, 829-839. doi: 10.1038/nrn1201

Bechara, A., Damasio, H., Tranel, D., and Anderson, S. W. (1998). Dissociation of working memory from decision making within the human prefrontal cortex. J. Neurosci. 18, 428-437.

Bull, R., Espy, K. A., and Wiebe, S. A. (2008). Short-term memory, working memory, and executive functioning in preschoolers: longitudinal predictors of mathematical achievement at age 7 years. Dev. Neuropsychol. 33, 205-228. doi: 10.1080/87565640801982312

Chaddock, L., Erickson, K. I., Prakash, R. S., Kim, J. S., Voss, M. W., VanPatter, M., et al. (2010). A neuroimaging investigation of the association between aerobic fitness, hippocampal volume, and memory performance in preadolescent children. Brain Res. 1358, 172-183. doi: 10.1016/j.brainres.2010.08.049

Chang, Y. K., Labban, J. D., Gapin, J. I., and Etnier, J. L. (2012). The effects of acute exercise on cognitive performance: a meta-analysis. Brain Res. 1453, 87-101. doi: 10.1016/j.brainres.2012.02.068

Chang, Y. K., Tsai, Y. J., Chen, T. T., and Hung, T. M. (2013). The impacts of coordinative exercise on executive function in kindergarten children: an ERP study. Exp. Brain Res. 225, 187-196. doi: 10.1007/s00221-012-3360-9

Chapman, S. B., Aslan, S., Spence, J. S., DeFina, L. F., Keebler, M. W., Didehbani, N., et al. (2013). Shorter term aerobic exercise improves brain, cognition, and cardiovascular fitness in aging. Front. Aging Neurosci. 5:75. doi: 10.3389/fnagi.2013.00075

Chen, A. G., Yan, J., Yin, H. C., Pan, C. Y., and Chang, Y. K. (2014). Effects of acute aerobic exercise on multiple aspects of executive function in preadolescent children. Psychol. Sport Exerc. 15, 627-636. doi: 10.1016/j.psychsport.2014.06.004

Clower, D. M., West, R. A., Lynch, J. C., and Strick, P. L. (2001). The inferior parietal lobule is the target of output from the superior colliculus, hippocampus, and cerebellum. J. Neurosci. 21, 6283-6291.

Cohen, J. D., Perlstein, W. M., Braver, T. S., Nystrom, L. E., Noll, D. C., Jonides, J., et al. (1997). Temporal dynamics of brain activation during a working memory task. Nature 386, 604-608. doi: 10.1038/386604a0

Constantinidis, C., and Klingberg, T. (2016). The neuroscience of working memory capacity and training. Nat. Rev. Neurosci. 17, 438-449. doi: 10.1038/nrn. 2016.43

Culham, J. C., and Kanwisher, N. G. (2001). Neuroimaging of cognitive functions in human parietal cortex. Curr. Opin. Neurobiol. 11, 157-163. doi: 10.1016/S09594388(00)00191-4

Darki, F., and Klingberg, T. (2014). The role of fronto-parietal and fronto-striatal networks in the development of working memory: a longitudinal study. Cereb. Cortex 25, 1587-1595. doi: 10.1093/cercor/bht352

Davis, C. L., Tomporowski, P. D., Boyle, C. A., Waller, J. L., Miller, P. H., Naglieri, J. A., et al. (2007). Effects of aerobic exercise on overweight children's cognitive functioning: a randomized controlled trial. Res. Q. Exerc. Sport 78, 510-519. doi: 10.5641/193250307X13082512817660

Davis, C. L., Tomporowski, P. D., McDowell, J. E., Austin, B. P., Miller, P. H., Yanasak, N. E., et al. (2011). Exercise improves executive function and achievement and alters brain activation in overweight children: a randomized, controlled trial. Health Psychol. 30, 91-98. doi: 10.1037/a002 1766

de Jong, P. F. (1998). Working memory deficits of reading disabled children. J. Exp. Child Psychol. 70, 75-96. doi: 10.1006/jecp.1998.2451
Diamond, A., and Lee, K. (2011). Interventions shown to aid executive function development in children 4 to 12 years old. Science 333, 959-964. doi: 10.1126/science. 1204529

Diwadkar, V. A., Carpenter, P. A., and Just, M. A. (2000). Collaborative activity between parietal and dorso-lateral prefrontal cortex in dynamic spatial working memory revealed by fMRI. Neuroimage 12, 85-99. doi: 10.1006/nimg.2000.0586

Drollette, E. S., Scudder, M. R., Raine, L. B., Moore, R. D., Saliba, B. J., Pontifex, M. B., et al. (2014). Acute exercise facilitates brain function and cognition in children who need it most: an ERP study of individual differences in inhibitory control capacity. Dev. Cogn. Neurosci. 7, 53-64. doi: 10.1016/j.dcn.2013.11.001

Ericsson, K. A., and Kintsch, W. (1995). Long-term working memory. Psychol. Rev. 102, 211-245. doi: 10.1037/0033-295X.102.2.211

Erickson, K. I., and Kramer, A. F. (2009). Aerobic exercise effects on cognitive and neural plasticity in older adults. Br. J. Sports Med. 43, 22-24. doi: 10.1136/bjsm.2008.052498

Erickson, K. I., Voss, M. W., Prakash, R. S., Basak, C., Szabo, A., Chaddock, L., et al. (2011). Exercise training increases size of hippocampus and improves memory. Proc. Natl. Acad. Sci. U.S.A. 108, 3017-3022. doi: 10.1073/pnas.1015950108

Ester, E. F., Sprague, T. C., and Serences, J. T. (2015). Parietal and frontal cortex encode stimulus-specific mnemonic representations during visual working memory. Neuron 87, 893-905. doi: 10.1016/j.neuron.2015.07.013

Fox, M. D., and Raichle, M. E. (2007). Spontaneous fluctuations in brain activity observed with functional magnetic resonance imaging. Nat. Rev. Neurosci. 8, 700-711. doi: 10.1038/nrn2201

Harding, I. H., Yücel, M., Harrison, B. J., Pantelis, C., and Breakspear, M. (2015). Effective connectivity within the frontoparietal control network differentiates cognitive control and working memory. Neuroimage 106, 144-153. doi: 10.1016/j.neuroimage.2014.11.039

Hillman, C. H., Erickson, K. I., and Kramer, A. F. (2008). Be smart, exercise your heart: exercise effects on brain and cognition. Nat. Rev. Neurosci. 9, 58-65. doi: $10.1038 / \mathrm{nrn} 2298$

Hillman, C. H., Pontifex, M. B., Raine, L. B., Castelli, D. M., Hall, E. E., and Kramer, A. F. (2009). The effect of acute treadmill walking on cognitive control and academic achievement in preadolescent children. Neuroscience 159, 1044-1054. doi: 10.1016/j.neuroscience.2009.01.057

Hoekzema, E., Carmona, S., Tremols, V., Gispert, J. D., Guitart, M., Fauquet, J., et al. (2010). Enhanced neural activity in frontal and cerebellar circuits after cognitive training in children with attention-deficit/hyperactivity disorder. Hum. Brain Mapp. 31, 1942-1950. doi: 10.1002/hbm.20988

Hötting, K., and Röder, B. (2013). Beneficial effects of physical exercise on neuroplasticity and cognition. Neurosci. Biobehav. Rev. 37, 2243-2257. doi: 10.1016/j.neubiorev.2013.04.005

Jonides, J., Schumacher, E. H., Smith, E. E., Koeppe, R. A., Awh, E., Reuter-Lorenz, P. A., et al. (1998). The role of parietal cortex in verbal working memory. J. Neurosci. 18, 5026-5034.

Kamijo, K., Pontifex, M. B., O'Leary, K. C., Scudder, M. R., Wu, C. T., Castelli, D. M., et al. (2011). The effects of an afterschool physical activity program on working memory in preadolescent children. Dev. Sci. 14, 1046-1058. doi: 10.1111/j.1467-7687.2011.01054.x

Klingberg, T. (2006). Development of a superior frontal-intraparietal network for visuo-spatial working memory. Neuropsychologia 44, 2171-2177. doi: 10.1016/j.neuropsychologia.2005.11.019

Klingberg, T., Forssberg, H., and Westerberg, H. (2002). Increased brain activity in frontal and parietal cortex underlies the development of visuospatial working memory capacity during childhood. J. Cogn. Neurosci. 14, 1-10. doi: $10.1162 / 089892902317205276$

Koenigs, M., Barbey, A. K., Postle, B. R., and Grafman, J. (2009). Superior parietal cortex is critical for the manipulation of information in working memory. J. Neurosci. 29, 14980-14986. doi: 10.1523/JNEUROSCI.3706-09.2009

Kramer, A. F., and Erickson, K. I. (2007). Capitalizing on cortical plasticity: influence of physical activity on cognition and brain function. Trends Cogn. Sci. 11, 342-348. doi: 10.1016/j.tics.2007.06.009

LaBar, K. S., Gitelman, D. R., Parrish, T. B., and Mesulam, M. M. (1999) Neuroanatomic overlap of working memory and spatial attention networks: a functional MRI comparison within subjects. Neuroimage 10, 695-704. doi: 10.1006/nimg.1999.0503

Lardon, M. T., and Polich, J. (1996). EEG changes from long-term physical exercise. Biol. Psychol. 44, 19-30. doi: 10.1016/S0301-0511(96)05198-8 
Li, L., Men, W. W., Chang, Y. K., Fan, M. X., Ji, L., and Wei, G. X. (2014). Acute aerobic exercise increases cortical activity during working memory: a functional MRI study in female college students. PLOS ONE 9:e99222. doi: 10.1371/journal.pone.0099222

McMorris, T., Sproule, J., Turner, A., and Hale, B. J. (2011). Acute, intermediate intensity exercise, and speed and accuracy in working memory tasks: a meta-analytical comparison of effects. Physiol. Behav. 102, 421-428. doi: 10.1016/j.physbeh.2010.12.007

Mrazek, M. D., Franklin, M. S., Phillips, D. T., Baird, B., and Schooler, J. W. (2013). Mindfulness training improves working memory capacity and GRE performance while reducing mind wandering. Psychol. Sci. 24, 776-781. doi: $10.1177 / 0956797612459659$

Oldfield, R. C. (1971). The assessment and analysis of handedness: the Edinburgh inventory. Neuropsychologia 9, 97-113. doi: 10.1016/0028-3932(71)90067-4

Olesen, P. J., Westerberg, H., and Klingberg, T. (2004). Increased prefrontal and parietal activity after training of working memory. Nat. Neurosci. 7, 75-79. doi: 10.1038/nn1165

Osaka, N., Osaka, M., Kondo, H., Morishita, M., Fukuyama, H., and Shibasaki, H. (2004). The neural basis of executive function in working memory: an fMRI study based on individual differences. Neuroimage 21, 623-631. doi: 10.1016/j.neuroimage.2003.09.069

Owen, A. M., McMillan, K. M., Laird, A. R., and Bullmore, E. (2005). $\mathrm{N}$-back working memory paradigm: a meta-analysis of normative functional neuroimaging studies. Hum. Brain Mapp. 25, 46-59. doi: 10.1002/hbm.20131

Passolunghi, M. C., and Siegel, L. S. (2001). Short-term memory, working memory, and inhibitory control in children with difficulties in arithmetic problem solving. J. Exp. Child Psychol. 80, 44-57. doi: 10.1006/jecp.2000.2626

Pontifex, M., Hillman, C., Fernhall, B. O., Thompson, K., and Valentini, T. (2009). The effect of acute aerobic and resistance exercise on working memory. Med. Sci. Sports Exerc. 41, 927-934. doi: 10.1249/MSS.0b013e3181907d69

Siegel, L. S., and Ryan, E. B. (1989). The development of working memory in normally achieving and subtypes of learning disabled children. Child Dev. 60, 973-980. doi: 10.2307/1131037

Smith, E. E., and Jonides, J. (1997). Working memory: a view from neuroimaging. Cogn. Psychol. 33, 5-42. doi: 10.1006/cogp.1997.0658

Smith, E. E., and Jonides, J. (1999). Storage and executive processes in the frontal lobes. Science 283, 1657-1661. doi: 10.1126/science.283.5408.1657

Stoodley, C. J., and Schmahmann, J. D. (2009). Functional topography in the human cerebellum: a meta-analysis of neuroimaging studies. Neuroimage 44, 489-501. doi: 10.1016/j.neuroimage.2008.08.039
Swanson, H. L., and Sachse-Lee, C. (2001). Mathematical problem solving and working memory in children with learning disabilities: both executive and phonological processes are important. J. Exp. Child Psychol. 79, 294-321. doi: 10.1006/jecp.2000.2587

Takeuchi, H., Sekiguchi, A., Taki, Y., Yokoyama, S., Yomogida, Y., Komuro, N., et al. (2010). Training of working memory impacts structural connectivity. J. Neurosci. 30, 3297-3303. doi: 10.1523/JNEUROSCI.4611-09. 2010

Todd, J. J., and Marois, R. (2005). Posterior parietal cortex activity predicts individual differences in visual short-term memory capacity. Cogn. Affect. Behav. Neurosci. 5, 144-155. doi: 10.3758/CABN.5.2.144

Verburgh, L., Königs, M., Scherder, E. J., and Oosterlaan, J. (2013). Physical exercise and executive functions in preadolescent children, adolescents and young adults: a meta-analysis. Br. J. Sports Med. 48, 973-979. doi: 10.1136/bjsports2012-091441

Vinogradova, O. S. (2001). Hippocampus as comparator: role of the two input and two output systems of the hippocampus in selection and registration of information. Hippocampus 11, 578-598. doi: 10.1002/hipo.1073

Vogel, E. K., and Machizawa, M. G. (2004). Neural activity predicts individual differences in visual working memory capacity. Nature 428, 748-751. doi: 10.1038 /nature 02447

Voss, M. W., Erickson, K. I., Prakash, R. S., Chaddock, L., Kim, J. S., Alves, H., et al. (2013). Neurobiological markers of exercise-related brain plasticity in older adults. Brain Behav. Immun. 28, 90-99. doi: 10.1016/j.bbi.2012.10.021

Westerberg, H., and Klingberg, T. (2007). Changes in cortical activity after training of working memory-a single-subject analysis. Physiol. Behav. 92, 186-192. doi: 10.1016/j.physbeh.2007.05.041

Zhang, H. (2009). The revision of WISC-IV Chinese version. Psychol. Sci. 32, $1177-1179$.

Conflict of Interest Statement: The authors declare that the research was conducted in the absence of any commercial or financial relationships that could be construed as a potential conflict of interest.

Copyright $\odot 2016$ Chen, Zhu, Yan and Yin. This is an open-access article distributed under the terms of the Creative Commons Attribution License (CC BY). The use, distribution or reproduction in other forums is permitted, provided the original author(s) or licensor are credited and that the original publication in this journal is cited, in accordance with accepted academic practice. No use, distribution or reproduction is permitted which does not comply with these terms. 